\title{
Measurement of Involute Profile with Small Clamping Eccentricity in a Gear Measuring Center
}

\author{
Han Lianfu $1,2, *$ - Tang Wenyan ${ }^{1}$ - Wang Jun ${ }^{1}$ - Fu Changfeng 2 \\ ${ }^{1}$ Harbin Institute of Technology, Institute of Precision Instrument, China \\ 2 Northeast Petroleum University, School of Electronic Science, China
}

\begin{abstract}
This paper studies the influence of small clamping eccentricity on the measurement and evaluation of involute profile, analyzes the cause of clamping eccentric error, establishes a clamping eccentric error compensation model, and introduces a computing method of clamping eccentric parameters. Experimental testing of the computing method of clamping eccentric parameters proves that the computing method of clamping eccentric parameters is correct and feasible when the clamping eccentric value is within three quarters of the measurement range of the probe. The results of the clamping eccentricity error compensation experiment show that the clamping eccentric error compensation model can compensate measurement results to the results attained under operating conditions, and achieve precision measurement of involute profiles. The highest allowed clamping eccentric value can be extended to three quarters of the measurement range of the probe by the clamping eccentricity error compensation model proposed in this paper, thereby saving time otherwise spent adjusting for clamping eccentricity.
\end{abstract}

Keywords: gear measuring center, clamping eccentricity, involute profile, measurement, evaluation

\section{INTRODUCTION}

Involute gears are widely used in mechanical manufacturing, and the quality of the involute profile affects gear vibration and noise [1] and [2]. Measurement of involute profile is difficult, and the involute profile is usually measured by a gear measuring center [3] and [4]. Their measuring principle is the Electronic-generating method, which demands that the rotation of the center axis of the gear measuring center should coincide with the geometric center line of the gear [5] to [7]. It is easy to meet the clamping demand for middle and small size external gears with a core shaft and special clamp. But internal gears cannot be clamped on the measurement platform with a core shaft and special clamp because there is no position to clamp internal gears. Large gears also cannot be clamped on the measurement platform because they are too large to find a proper core shaft [7]. Large and internal gears are clamped on the measurement platform of the gear measuring center using a number of magnets. In the clamping process of large gears and internal gears, a manual method with a dial indicator is involved to assist in the clamping. Therefore, the geometric center line of the gear often parallel deviates from the rotation center axis of the gear measuring center due to the limitations of manual micro-positioning ability and the accuracy of the auxiliary clamping instrument, resulting in clamping eccentricity, where the error caused by this is called the clamping eccentric error.

The clamping eccentric error inevitably reduces the measurement accuracy of gear measuring center. To decrease the influence of the clamping eccentricity on the measurement of the involute profile, the value of the clamping eccentricity is adjusted to be as small as possible, but this takes a lot of time and the clamping eccentricity still exists [8]. Therefore, accurate measurement of the involute profile with clamping eccentricity is a real problem. A method of compensating for clamping eccentricity error was proposed by Yang, and the method achieves clamping eccentric error compensation by correcting the transformation of the workpiece coordinate system and the instrument coordinate system. However, this method needs specially designed measurement nets to obtain the proper clamping eccentricity parameters [9]. Shi proposes a method to compensate for clamping eccentricity [10], which measures the net points on the involute profile and uses the least square method to estimate the clamping eccentricity parameters, but the method needs to include an extra measurement trajectory for the computation of eccentricity parameters. At present, the gear measuring center made by Klingelnberg allows the measurement of involute profile with clamping eccentricity. The allowed maximum eccentricity value is $2 \mathrm{~mm}$ and the eccentric parameters are obtained by measurement of the core shaft. However, its disadvantage is its inaccessibility to internal and large gears [11].

This paper proposes a measurement method for involute profiles with small clamping eccentricity for internal gears and large gears based on a gear measuring center. The method not only allows precision measurement of involute profiles without changing the measurement trajectory, but also reduces the time needed to adjust the clamping eccentricity. 


\section{THE INFLUENCE OF CLAMPING ECCENTICITY ON MEASUREMENT AND EVALUATION OF INVOLUTE PROFILE}

To measure the involute profile with a small clamping eccentricity, the influence of the clamping eccentricity on the measurement and evaluation of the involute profile should be considered. When there is no clamping eccentricity, the measurement trajectory of the probe relative to the gear is an involute curve attained through the linkage of the $X$-axis and main shaft. If the $X$-axis and main shaft still maintain their linkage in the default way in the clamping eccentric state, the profiles deviate from their default measurement position by the existence of the clamping eccentricity. So, any point $P_{0}\left(x_{0}, y_{0}, z_{0}\right)$ attained by generating the measured values of involute profiles deviates from its default measurement position, as shown in Fig. 1a. The measured points are in the same plane, because the $Z$-axis remains static when the involute profile is measured. Although there is clamping eccentricity, the measured curve is part of the involute curve to be measured and the point $P_{0}\left(x_{0}, y_{0}, z_{0}\right)$ is still in the curve to be measured. The point $P_{0}\left(x_{0}, y_{0}, z_{0}\right)$ may delay or advance its actual position, and it may appear at position $P_{1}$-point or $P_{2}$-point, both of which are on the measured involute profile, as shown in Fig. 1b.

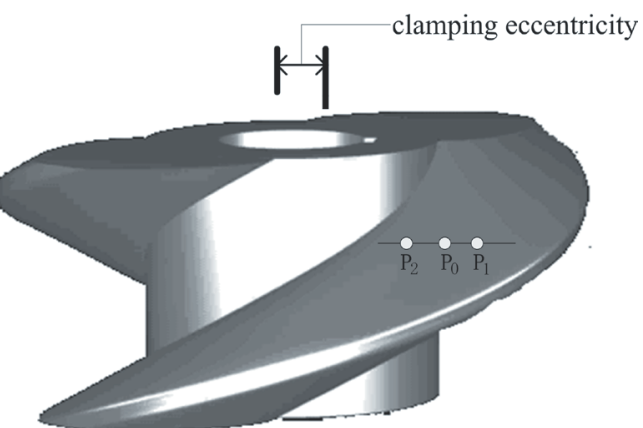

the rotation center axis of : the geometric center a) gear measuring center l line of gear

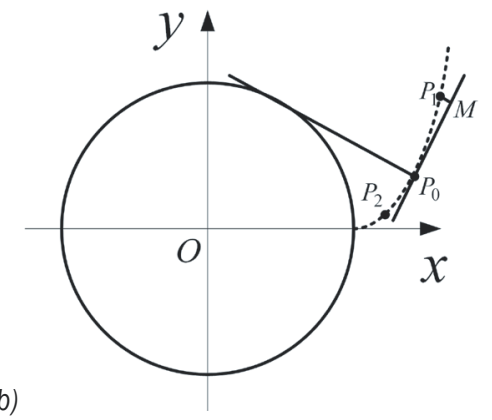

Fig. 1. The influence of clamping eccentricity on measurement and evaluation of the involute profile; a) gear in the clamping eccentricity condition, and b) the schematic diagram of a section
It is important to study the usage of the above rule in measuring the involute profile with clamping eccentricity without changing the measurement trajectory. It is assumed that involute profiles are by default in their measurement position during the process of measurement of involute profiles in the clamping eccentric measurement state, so the probe may impact the gear if the clamping eccentricity is too large. The measurement range of the probe is $L$, so the maximum allowed deviation $\Delta d_{\max }$ between the rotation center of the instrument and the geometric center of the gear is expressed as:

$$
\Delta d_{\max }=L
$$

The output and input of the probe have a strictly linear relationship within three quarters of the measurement range of the probe, so the appropriate maximum value of the clamping eccentric value $\Delta e$ is given by Eq. (2).

$$
\Delta e=0.75 \mathrm{~L} .
$$

For all kinds of gears, except for micro gears, the relation between $d_{1}$ (the distance from the starting point of evaluation to the starting point of measurement) and $d_{2}$ (the distance from the end point of evaluation to the end point of measurement) can be expressed as:

$$
\left\{\begin{array}{l}
d_{1} \approx 0.5\left(L_{A E}-L_{\alpha}\right)>>\Delta e=0.75 L \\
d_{2} \approx 0.5\left(L_{A E}-L_{\alpha}\right)>>\Delta e=0.75 L
\end{array}\right.
$$

where, $L_{A E}$ is the abbreviation of active length and $L_{\alpha}$ is the abbreviation of the profile evaluation range [12].

According to Eq.(3), the length of the profile that is not evaluated is much larger than the clamping eccentric value. The clamping eccentricity only affects the lengths of $d_{1}$ and $d_{2}$, and the evaluation length is not affected when the clamping eccentricity is within $0.75 \mathrm{~L}$. So, the part of the curve involved in the evaluation can be measured without adjusting the measurement trajectory, but the profiles deviate from their default measurement position due to the existence of the clamping eccentricity. As shown in Fig. $1 \mathrm{~b}$, the measurement values that stand for the $P_{0^{-}}$ point in the operation condition stand for the $P_{1}$-point in the clamping eccentricity condition, an error of $\overline{P_{1} M}$ is caused during the total deviation computation of the tooth profile. The clamping eccentricity error should be compensated for in the evaluation process of involute profiles, although the measurement trajectory of involute profile does not need to be changed when the clamping eccentric value is $0.75 \mathrm{~L}$. 


\section{2 "THE CLAMPING ECCENTRIC ERROR COMPENSATION MODEL}

\subsection{Cause of the Clamping Eccentric Error}

To establish the clamping eccentric error compensation model, the cause of the clamping eccentric error should be studied. The coordinates of involute profiles in the workpiece coordinate system should be transformed to the instrument coordinate system. While evaluating involute profiles, the measured values in the instrument coordinate system should be transformed into the workpiece coordinate system. Therefore, it is necessary to study the transformational relationship between these two coordinate systems. The workpiece coordinate system is established with the geometrical center of the gear as the origin, and the $X, Y$ and $Z$ directions of the instrument coordinate system as $X_{0}, Y_{0}$ and $Z_{0}$ directions of the workpiece coordinate system, as shown in Fig. 2. The geometric center line of the gear parallel deviates from the rotation center axis of gear measuring center in the clamping eccentric state and the gear is clamped onto the platform of a gear measuring center, the origin of workpiece coordinate system in the instrument coordinate system keeps changing and it is denoted as $O_{0 i}$.

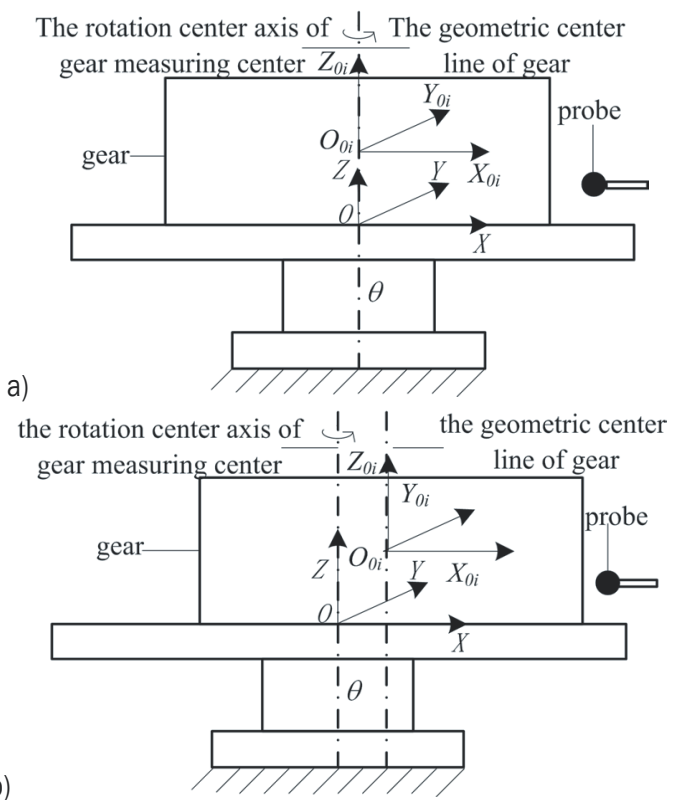

Fig. 2. Construction of the workpiece coordinate system; a) in the ideal state, and b) in the clamping eccentric state

As shown in Fig. 2a, the transformational relationship of the coordinates in the ideal state is expressed as:

$$
\left[\begin{array}{c}
x_{i} \\
y_{i} \\
z_{i}
\end{array}\right]=\left[\begin{array}{l}
x_{0 i_{i}} \\
y_{0 i_{i}} \\
z_{0 i_{i}}
\end{array}\right]+\left[\begin{array}{l}
0 \\
0 \\
\frac{b}{2}
\end{array}\right]=\left[\begin{array}{c}
x_{0 i_{i}} \\
y_{0 i_{i}} \\
z_{0 i_{i}}
\end{array}\right]+\alpha,
$$

where $b$ is the face width, and $\alpha$ is the translation vector of the $Z$ direction between the workpiece coordinate system and the instrument coordinate system in the ideal state.

As shown in Fig. $2 b$, the transformational relationship of the coordinates in the clamping eccentric state can be expressed as:

$$
\left[\begin{array}{c}
x_{i} \\
y_{i} \\
z_{i}
\end{array}\right]=\left[\begin{array}{c}
x_{0 i_{i}} \\
y_{0 i_{i}} \\
z_{0 i_{i}}
\end{array}\right]+\left[\begin{array}{c}
0 \\
0 \\
\frac{b}{2}
\end{array}\right]+\left[\begin{array}{c}
m_{i} \\
n_{i} \\
0
\end{array}\right]=\left[\begin{array}{c}
x_{0 i_{i}} \\
y_{0 i_{i}} \\
z_{0 i_{i}}
\end{array}\right]+\alpha+\beta_{i},
$$

where $\beta_{i}$ is the clamping eccentricity transformational vector between the workpiece coordinate system and the instrument coordinate system, and $\left(m_{i}, n_{i}, b / 2\right)$ is the origin of the workpiece coordinate system in the instrument coordinate system.

When the clamping eccentric value is small, $\beta_{i} \approx 0$ and Eq. (5) is approximately valid if $\beta_{i}$ is neglected. Under this condition, the influence of neglecting $\beta_{i}$ on the transformational accuracy of the coordinates is tiny and the clamping eccentric error can be neglected. When the clamping eccentric value is large, $\beta_{i}>0$ and Eq. (5) is not valid if $\beta_{i}$ is neglected. The influence of neglecting $\beta_{i}$ on the transformational accuracy of the coordinates is large, so it would result in a large transformational error of the coordinates. Under this condition, the clamping eccentric error should not be neglected, but in fact the clamping eccentricity transformational vector $\beta_{i}$ is still not considered, so the clamping eccentric error appears. From the preceding analysis it is clear that the clamping eccentric error is caused by the transformation error between the workpiece coordinate system and the instrument coordinate system due to ignoring the clamping eccentricity transformational vector $\beta_{i}$.

\subsection{Establishment of the Clamping Eccentric Error Compensation Model}

According to Eq. (5), $\beta_{i}$ is determined by the origin $O_{0 i}\left(m_{i}, n_{i}\right)$ of the workpiece coordinate system in the instrument coordinate system. Because the gear is clamped on the measurement platform of the gear measuring center, the distance between the origin of the workpiece coordinate system and the origin of 
the instrument coordinate system is a constant. When the gear revolves $2 \pi$ around the rotation center axis of gear measuring center, the geometric center of the gear moves in circle $O$, as shown in Fig. 3. It is assumed that the origin of workpiece coordinate system at a certain measurement time is at the position point $O_{00}$, the clamping eccentric value that the initial position of the gear geometrical center axis deviates from the rotation center axis is $e_{0}=e$, and the polar angle is $\phi_{0}$ and the reading of main shaft is $\theta_{0}$. After main shaft rotates $\theta_{i}$, the origin of the workpiece coordinate system revolves to point $O_{0 i}$. As shown in Fig. 3, the eccentric value $e_{i}$ and the polar angle $\phi_{i}$ at this measurement moment can be obtained as:

$$
\left\{\begin{array}{l}
e_{i}=e \\
\varphi_{i}=\varphi_{0}+\theta_{i}
\end{array},\right.
$$

where $\theta_{i}$ is the angle that the main shaft rotates to the initial angle $\theta_{0}$.

The origin of the workpiece coordinate system in the instrument coordinate system is point $O_{0 i}\left(m_{i}, n_{i}\right)$, and the equations of the orgin are as follows:

$$
\left\{\begin{array}{c}
m_{i}=e \cos \varphi_{i}=e \cos \left(\varphi_{0}+\theta_{i}\right) \\
n_{i}=e \sin \varphi_{i}=e \sin \left(\varphi_{0}+\theta_{i}\right)
\end{array} .\right.
$$

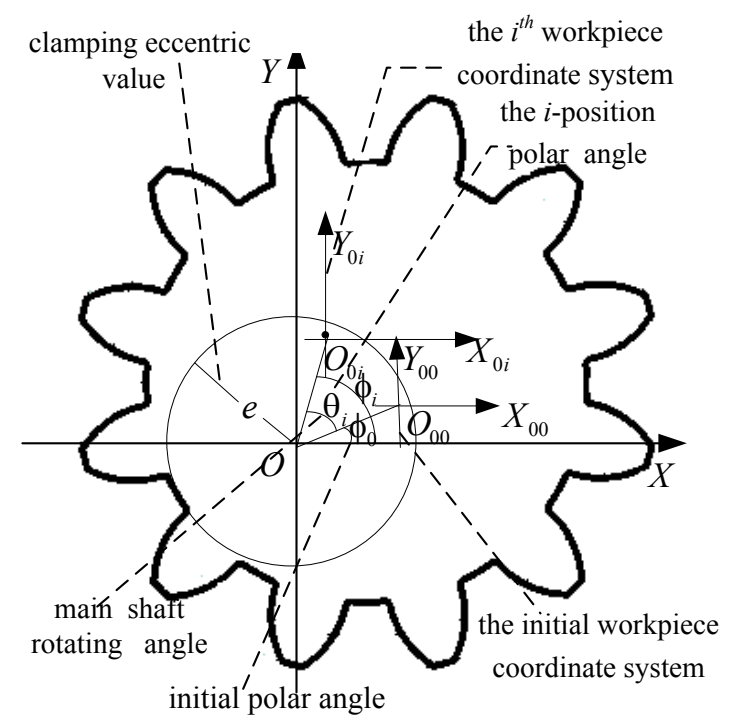

Fig. 3. Running track of the gear's geometrical center

When the origin of the workpiece coordinate system is at the position point $O_{0 i}$, the workpiece coordinate system is denoted as the $i^{\text {th }}$ workpiece coordinate system. For any point $P_{i}\left(x_{i}, y_{i}, z_{i}, \theta_{i}\right)$ in the instrument coordinate system, according to Eqs. (2) and (7), the coordinate transformational relationship between the instrument coordinate system and the $i$-position of workpiece coordinate system is expressed as Eq.(8):

$$
\left[\begin{array}{c}
x_{i} \\
y_{i} \\
z_{i}
\end{array}\right]=\left[\begin{array}{c}
x_{0 i_{i}} \\
y_{0 i_{i}} \\
z_{0 i_{i}}
\end{array}\right]+\alpha+\left[\begin{array}{c}
e \cos \left(\varphi_{0}+\theta_{i}\right) \\
e \sin \left(\varphi_{0}+\theta_{i}\right) \\
0
\end{array}\right] .
$$

The measured values of involute profiles should be transferred into the same workpiece coordinate system in the evaluation. In this paper, the measured values are transfered into the initial workpiece coordinate system. As shown in Fig. 3, the coordinate transformational relationship between the initial workpiece coordinate system and the $i$-position of workpiece coordinate system can be expressed as:

$$
\left[\begin{array}{c}
x_{00_{i}} \\
y_{00_{i}} \\
z_{00_{i}} \\
1
\end{array}\right]=M\left[\begin{array}{c}
x_{0 i_{i}} \\
y_{0 i_{i}} \\
z_{0 i_{i}} \\
1
\end{array}\right]
$$

where, $M$ is the coordinate transformational matrix between the initial workpiece coordinate system and the $i$-position of the workpiece coordinate system and it is expressed as:

$$
M=\left[\begin{array}{cccc}
1 & 0 & 0 & -e\left[\cos \phi_{0}-\cos \left(\phi_{0}+\theta_{i}\right)\right] \\
0 & 1 & 0 & e\left[\sin \left(\phi_{0}+\theta_{i}\right)-\sin \phi_{0}\right] \\
0 & 0 & 1 & 0 \\
0 & 0 & 0 & 1
\end{array}\right],
$$

The clamping eccentric error compensation model is established according to Eqs. (8) and (9), and the expression of the model is given by Eq. (11).

$$
\left[\begin{array}{c}
x_{00_{i}} \\
y_{00_{i}} \\
z_{00_{i}} \\
1
\end{array}\right]=M\left[\begin{array}{c}
x_{i}-e \cos \left(\phi_{0}+\theta_{i}\right) \\
y_{i}-e \sin \left(\phi_{0}+\theta_{i}\right) \\
z_{i}-\frac{b}{2} \\
1
\end{array}\right] \text {. }
$$

Once the coordinate transformation without error between the initial workpiece coordinate system and the instrument coordinate system is realized by the clamping eccentric error compensation model, then the involute profiles can be evaluated according to the evaluation standard. According to Eq. (11), there are only two unknown parameters (clamping eccentric value $e$ and polar angle $\phi_{0}$ ) in the model. 


\subsection{Calculation of Clamping Eccentric Parameters e and $\phi_{0}$}

This part discusses the calculation of the clamping eccentric parameters according to the measuring points of the tooth profile. As shown in Fig. 4a, the probe is adjusted to the base circle in the $X$ direction. The proportional relationship between the rotation angle of the main shaft and the moving distance of the $Y$-guideway is determined by the parameters of the gear to be measured. The geometrical center $O_{00}\left(m_{0}, n_{0}\right)$ of the gear at a certain measurement moment is defined as the origin of the initial workpiece coordinate system, as shown in Fig. 4b.

a)
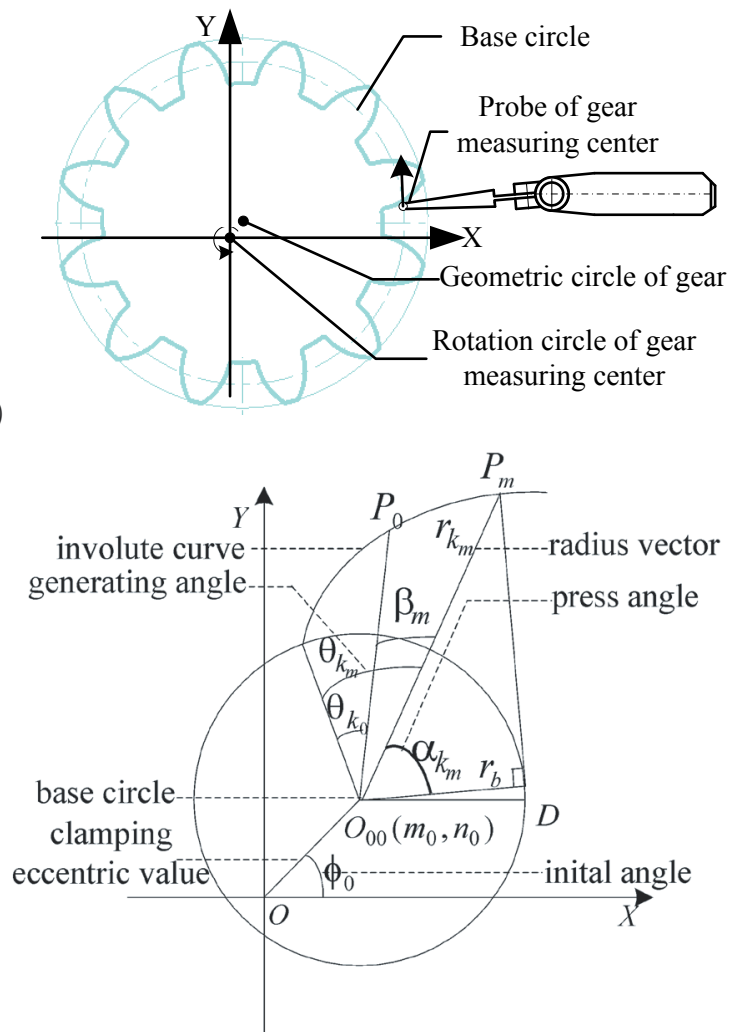

b)

Fig. 4. Determination of circle center using the involute curve; a) measurement of involute profiles, and b) example of an involute curve

The clamping eccentric angle $\phi_{0}$ and the clamping eccentric value $e$ can be expressed as:

$$
\left\{\begin{array}{c}
\phi_{0}=\angle O_{00} O X \\
e=\left|O_{00} O\right|
\end{array},\right.
$$

As shown in Fig. 4b, the involute curve $P_{0} P_{m}$ is the involute profile to be measured, and $O_{00}\left(\mathrm{~m}_{0}, n_{0}\right)$ is the center of the base circle and $r_{b}$ is the radius. The radius vector $r_{k_{m}}$ at the point $P_{m}\left(x_{m}, y_{m}\right)$ of the involute profile is expressed as:

$$
r_{k_{m}}=\sqrt{\left(x_{m}-m_{0}\right)^{2}+\left(y_{m}-n_{0}\right)^{2}} .
$$

As shown in Fig. 4b, involute curve $P_{0} P_{m}$ is the involute profile to be measured, and $O_{00}\left(\mathrm{~m}_{0}, n_{0}\right)$ is the center of the base circle and $r_{b}$ is the radius.

According to the characteristics of the involute, the expression for generating angle $\theta_{k_{m}}$ at the point $P_{m}\left(x_{m}, y_{m}\right)$ of the involute profile is as follows:

$$
\theta_{k_{m}}=\tan \alpha_{k_{m}}-\alpha_{k_{m}}
$$

where, $\alpha_{k_{m}}$ is the pressure angle at the point $P_{m}\left(x_{m}, y_{m}\right)$ of the involute profile and can be expressed as:

$$
\left\{\begin{array}{c}
\alpha_{k_{m}}=\arccos \frac{r_{b}}{r_{k_{m}}} \\
\tan \alpha_{k_{m}}=\frac{\sqrt{r_{k_{m}}^{2}-r_{b}^{2}}}{r_{b}} .
\end{array}\right.
$$

As shown in Fig. 4b, $\beta_{m}$ is expressed as Eq. (16):

$$
\begin{aligned}
\beta_{m} & =\angle D O_{00} P_{0}-\angle D O_{00} P_{m}= \\
& =\arctan \frac{y_{0}-n_{0}}{x_{0}-m_{0}}-\arctan \frac{y_{m}-n_{0}}{x_{m}-m_{0}} .
\end{aligned}
$$

As shown in Fig. $4 \mathrm{~b}, \beta_{m}$ can also be expressed as Eq. (17).

$$
\beta_{m}=\theta_{k_{m}}-\theta_{k_{0}}
$$

Let

$$
f_{m}=\theta_{k_{m}}-\theta_{k_{0}}-\angle D O_{00} P_{0}-\angle D O_{00} P_{m} .
$$

Eq. (19) should hold in theory, but it does not hold for the manufacturing error and measurement error.

$$
f_{m}=0 \text {. }
$$

According to the principle of the least square method, the following model for the identification of clamping eccentric parameters is established:

$$
\left\{\begin{array}{c}
F\left(m_{0}, n_{0}, r_{b}\right)=\min \sum_{m=1}^{N} f_{m}^{2} \\
\text { s.t } r_{b}>0
\end{array} .\right.
$$


Eq. (20) is a nonlinear least square problem and it can be solved by recursive methods [13]. In theory, as long as the measurement values of one tooth profile are obtained, $m_{0}$ and $n_{0}$ can be estimated. But it may result in a large deviation between the obtained eccentricity parameters and the true value. To reduce such a large deviation, the measurement values of teeth evenly distributed in the gear are adopted. The circle center is obtained from each tooth is $O_{0 i}\left(m_{0 i}, n_{0 i}\right)$. The optimum identification model of the circle center is established as follows:

$$
d\left(m_{0}, n_{0}\right)=\min \sum_{i=1}^{z_{0}}\left[\left(m_{0 i}-m_{0}\right)^{2}+\left(n_{0 i}-n_{0}\right)^{2}\right]
$$

where, $z_{0}$ is the measured number of teeth. The binary function $d\left(m_{0}, n_{0}\right)$ has its minimum value at the inflexion, so we take the partial derivative of $d\left(m_{0}, n_{0}\right)$ with respect to $m_{0}$ and $n_{0}$ to find the inflexion.

$$
\left\{\begin{array}{l}
\frac{\partial d\left(m_{0}, n_{0}\right)}{\partial m_{0}}=0 \\
\frac{\partial d\left(m_{0}, n_{0}\right)}{\partial n_{0}}=0
\end{array} .\right.
$$

The inflexion $\left(m_{0}, n_{0}\right)$, which is also the optimized circle center, can be obtained using Eq. (22). The clamping eccentric value e and the eccentric angle $\phi_{0}$ expressed as:

$$
\left\{\begin{array}{c}
\varphi_{0}=\arctan \left(n_{0} / m_{0}\right) \\
e=\sqrt{m_{0}^{2}+n_{0}{ }^{2}}
\end{array} .\right.
$$

\section{EXPERIMENTS}

To verify the correctness of the calculation method for clamping eccentricity parameters and the validity of the clamping eccentricity error compensation model, the following experiments were carried out with the internal gear on a L45 type gear measuring center. The measurement range of the probe in the experiment is $0.70 \mathrm{~mm}$ and the parameters of the internal gear are as follows: modulus is 3.25 ; tooth number is 60 ; modification coefficient is 0 ; face width is $30 \mathrm{~mm}$; pressure angle is $20^{\circ}$; spiral angle is $0^{\circ}$. Fig. 5 shows the measurement equipment. The gear is fixed on the platform with six magnets, the probe is adjusted to the base circle in the $X$ direction. The proportional relationship between the rotation angle of main shaft and the moving distance of the $Y$-guideway is determined by the parameters of the gear to be measured. The involute profile with small clamping eccentricity is compensated such that it is in the ideal state using the clamping eccentric error compensation model. Involute profiles are evaluated according to ISO1328-1 [12].

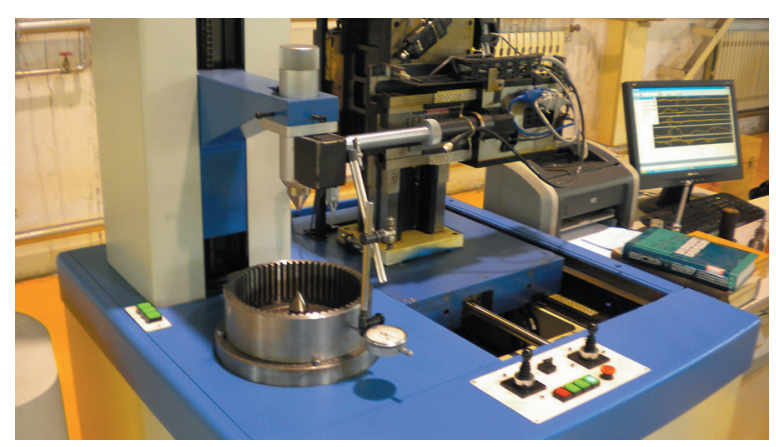

Fig. 5. The experimental equipment

\subsection{Experiment on the Calculation Method Of Clamping Eccentric Parameters}

The dial indicator is used to monitor the geometrical center position of the internal gear and to control the clamping eccentric value within $0.75 \mathrm{~L}$. The points of the addendum circle are measured every $0.5^{\circ}$ by the gear measuring center. As shown in Fig. $6, p_{i}\left(\mathrm{x}_{i}, \mathrm{y}_{i}\right)$ is one measurement point in the addendum circle, the circle center $o_{2}(\hat{a}, \hat{b})$ is obtained using the measured values of the points in the addendum circle using the Limacon Model [14].

$$
\left\{\begin{array}{l}
\hat{\mathrm{a}}=\frac{1}{\mathrm{~N}} \sum_{i=0}^{N-1} x_{i} \\
\hat{\mathrm{b}}=\frac{1}{\mathrm{~N}} \sum_{i=0}^{N-1} y_{i}
\end{array} .\right.
$$

The involute profiles of the gear are measured under the same clamping eccentric condition, and the center of base circle is obtained using the Involute Model proposed in this paper. To decrease contingency, a series of experiments were carried out under different clamping eccentric value conditions.

\subsection{The Clamping Eccentric Error Compensation Experiment}

Using the Involute Model proposed in this paper, the clamping eccentric value is $164.3 \mathrm{~mm}$ and the clamping eccentric angle is $43.62^{\circ}$. The clamping eccentric error compensation model is applied in the evaluation of the involute profiles. 


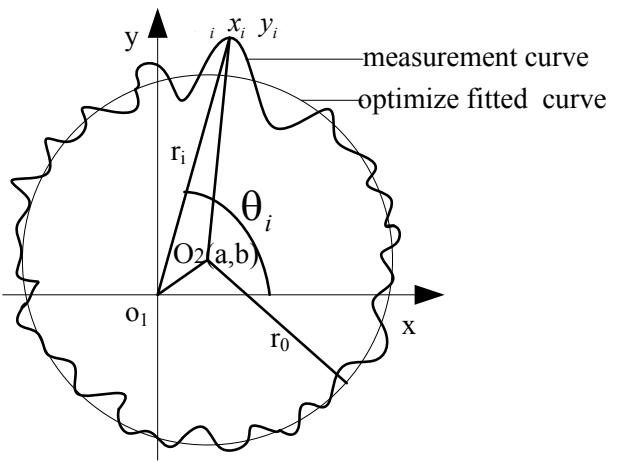

Fig. 6. Limacon Model

To decrease the influence of chance in the error compensation experiment, the position of the gear is adjusted and the involute profiles are measured 20 times for each clamping eccentric value. Then the preceding operation is repeated with a different clamping eccentric value.

\subsection{The Experiment of Saving Time in Using Adjusted Clamping Eccentricity}

To compare the saving time in using adjusted clamping eccentricity, a number of experiments were carried out with a series of gears whose tip diameter is less than $3000 \mathrm{~mm}$. To ensure the accuracy of the measurement, the clamping eccentric value is required to be as small as possible. For example, for a gear whose tip diameter is no more than $450 \mathrm{~mm}$, the clamping eccentric value is usually required to be less than $2 \mu \mathrm{m}$. The highest allowed clamping eccentric value can extend to 0.75 $L$ using the clamping eccentricity error compensation model proposed in this paper.

\section{RESULTS AND DISCUSSION}

\subsection{Results and Discussion of the Calculation Method of Clamping Eccentric Parameters}

In theory, the circle center of the addendum circle should coincide with the circle center of the base circle, and the clamping eccentric value and the clamping eccentric angle can be attained by the circle center according to Eq. (23). Table 1 shows a comparison of the results of the experiment on the calculation method of clamping eccentric parameters.

As shown in Table 1, both the eccentric values attained by Limacon Model and those attained by the Involute Model basically tally with those using the Indicator. The maximum deviation of clamping eccentric values is $1.9 \mathrm{~mm}$, which can be ignored compared to the maximum allowed clamping eccentric value of $0.75 \mathrm{~L}$. The maximal deviation of the eccentric angle between two models is $0.09^{\circ}$, which can also be ignored. This shows that the eccentricity parameter estimation method raised in this paper is correct and feasible.

\subsection{Results and Discussion of the Clamping Eccentric Error Compensation Experiment}

The measurement results before compensation are shown in Fig. 7a, then the clamping eccentric error compensation model is applied to the evaluation of the involute profiles and the deviation diagrams are shown in Fig. 7b.

The measurement results are shown in Table 2.

There are eight deviation curves of involute profiles in every deviation diagram in Fig. 7, and the deviation distance from the vertical lines in every curve stands for the deviation of the involute profiles. The deviation curves of involute profiles should be straight lines if there are no deviation in the profiles, and there is $1 \mathrm{~mm}$ per unit of profile deviation.. To display the deviation clearly, the deviations are enlarged 1000 times in Fig. 7. The numbers "1, 16, 31, 46 " mean the numbers of the measured tooth, and the numbers on the left stand for the number of left tooth profiles and those on the right stand for the number of right tooth profiles. Fig. $7 \mathrm{a}$ and $\mathrm{c}$ are obtained under the same measurement condition for the same four involute profiles. However, for the clamping eccentricity, the inclined degree of the deviation curves is obviously different, which indicates that $\pm \mathrm{fH} \alpha$ (profile slope deviation) increases greatly. The curve shape of the deviation curves is also different, which means that ffo (profile form deviation) and $\mathrm{F} \alpha$ (total profile deviation) are affected by the change in

Table 1. Estimated clamping eccentric parameters of different models

\begin{tabular}{lccccccccc}
\hline Limacon & $\mathrm{e}[\mu \mathrm{m}]$ & 29.3 & 69.1 & 100.4 & 164.4 & 228.5 & 299.8 & 357.2 & 491.7 \\
\cline { 2 - 11 } Model & $\Phi_{0}\left[^{\circ}\right]$ & 3.30 & 355.27 & 42.45 & 43.71 & 9.29 & 186.62 & 190.78 & 6.78 \\
\hline Involute & $e[\mu \mathrm{m}]$ & 29.1 & 68.7 & 98.5 & 164.3 & 227.2 & 298.7 & 356.1 & 490.3 \\
\cline { 2 - 11 } Model & $\Phi_{0}\left[^{\circ}\right]$ & 3.28 & 355.34 & 42.36 & 43.62 & 9.33 & 186.71 & 190.74 & 6.73 \\
\hline Dial Indicator & $e[\mu \mathrm{m}]$ & 30 & 70 & 100 & 160 & 230 & 300 & 360 & 490 \\
\hline
\end{tabular}


Table 2. Measurement results of involute profiles under different conditions

\begin{tabular}{lcccccc}
\hline & $F_{\alpha}[\mu \mathrm{m}]$ & Grade & $f_{f \alpha}[\mu \mathrm{m}]$ & Grade & $\pm f_{H \alpha}[\mu \mathrm{m}]$ & Grade \\
\hline Under operating conditions & 8.0 & 5 & 7.9 & 6 & -3.2 & 4 \\
\hline Before compensation & 35.7 & 9 & 37.2 & 10 & -17.8 & 9 \\
\hline After compensation & 8.3 & 5 & 7.6 & 6 & -3.1 & 4 \\
\hline
\end{tabular}

the evaluation position resulting from the clamping eccentricity. As shown in Table 2, due to clamping eccentricity, the 5 th grade $F \alpha$ is incorrectly judged as grade 9 , the 6 th grade $\mathrm{Ff} \alpha$ is incorrectly judged as grade 10 , and the 4 th grade $\pm \mathrm{fH} \alpha$ as grade 9 . Therefore, the measurement results are seriously affected. So, the measurement of tooth profile is seriously affected under conditiond of clamping eccentricity if the clamping eccentricity compensation is not taken into account. After compensation, the curves in Fig. 7b mostly accord with those in Fig. 7c in the evaluation range. According to Table 2, the measurement results after compensation are close to those under operating conditions and do not influence the evaluation grade.

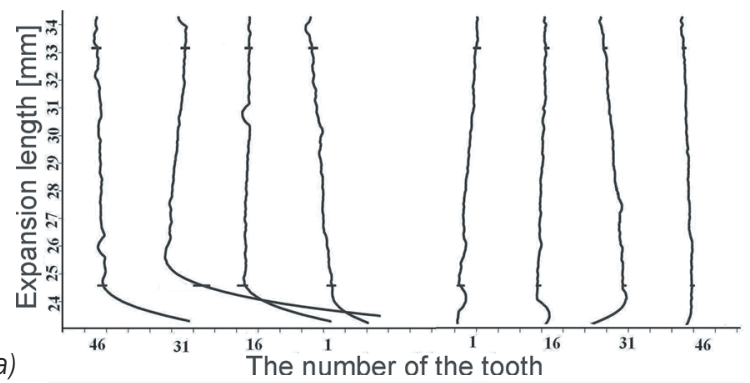

a)

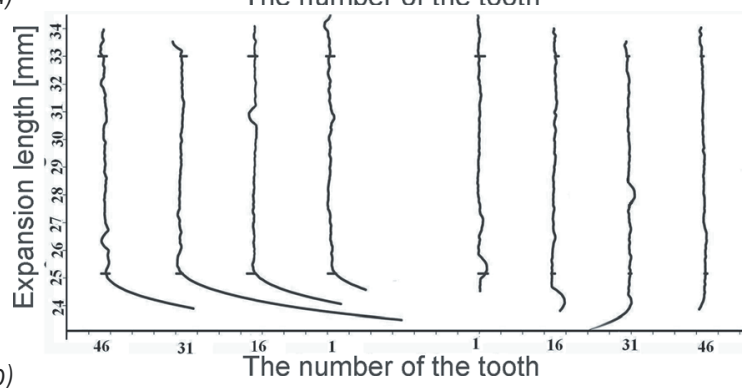

b)

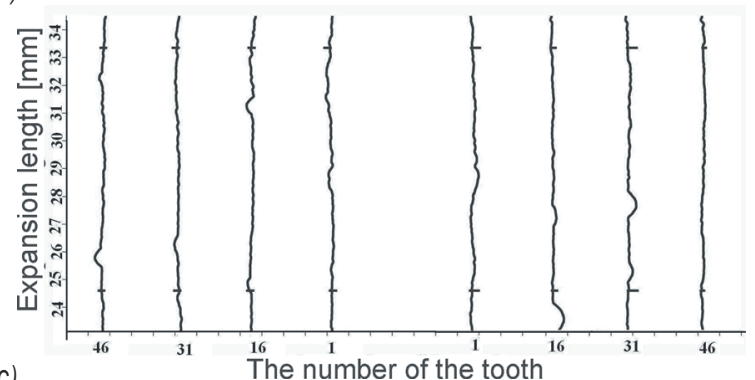

Fig. 7. Comparison of measurement results; a) the measurement result before compensation, b) the measurement result after compensation, c) the measurement result under operating conditions
The average measurement results of the involute profiles before and after compensation with different clamping eccentric values are shown in Fig. 8. As shown in Fig. 8, as the clamping eccentric value grows, the measurement errors of $F_{\alpha}, F_{f \alpha}$ and $\pm f_{H \alpha}$ all increase. The maximum measurement errors of $F_{\alpha}, F_{f \alpha}$ and $\pm f_{H \alpha}$ are, respectively, 31.6, 29.5 and $16.6 \mu \mathrm{m}$, so the measurement results seriously deviate from the true results under operating conditions.
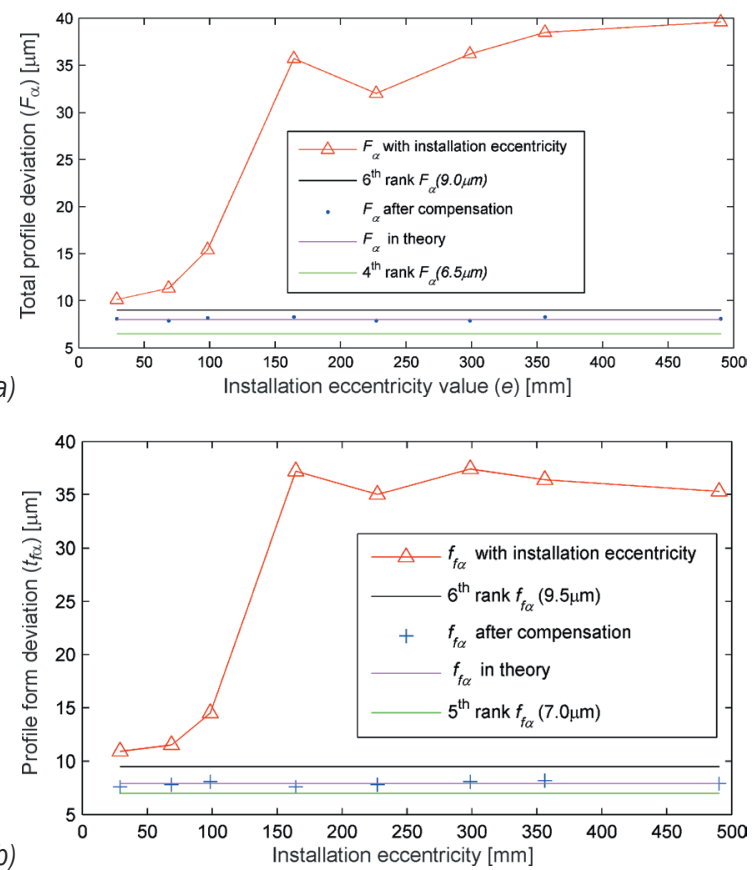

b)

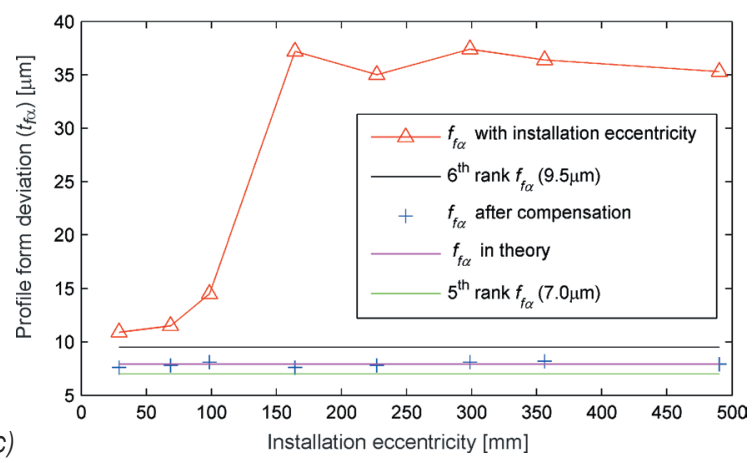

Fig. 8. Comparison of profile deviation before and after compensation; a) comparison of total profile deviation, b) comparison of profile form deviation, and c) comparison of profile slope deviation 
After compensation, the following measurement results are attained.

- The average value of $F_{\alpha}$ is $8.1 \mathrm{~mm}$, which approximates to the true value $8.0 \mathrm{~mm} . F_{\alpha}$ is the 5 th grade of accuracy class, which is also the same as the true grade.

- The average value of $F_{f \alpha}$ is $7.9 \mathrm{~mm}$, which approximates to the true value. $F_{f \alpha}$ is the 6 th grade, which is the same as the true grade.

- The average value of $\pm f_{H \alpha}$ is $-3.3 \mathrm{~mm}$, which approximates to the true value $-3.4 \mathrm{~mm} . \pm f_{H \alpha}$ is the $4^{\text {th }}$ grade of accuracy class, which is also the same as the true grade.

From the results of the preceding experiments, the measurement results after compensation basically tally with those obtained under operating conditions. Therefore, the clamping eccentricity error compensation model is correct.

\subsection{Results and Discussion of the saving time in adjusting the clamping eccentricity}

The highest allowed clamping eccentric value can extend to $0.75 \mathrm{~L}$ using the clamping eccentricity error compensation model proposed in this paper. The time saved adjusting the clamping eccentricity is shown in Fig. 9.

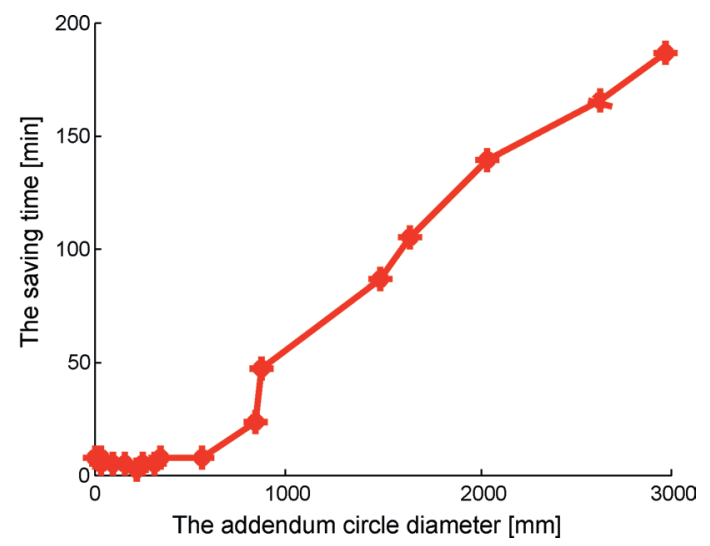

Fig. 9. Time saved adjusting clamping eccentricity

As shown in Fig.9, the time saved adjusting the clamping eccentricity increases with the increase of the addendum circle diameter. When the addendum circle diameter of a gear is within $600 \mathrm{~mm}$, the method can save five minutes, on average. When the addendum circle diameter of the gear is beyond $600 \mathrm{~mm}$, the time saved increases rapidly and the maximum time saved is 187 minutes. All in all, the method of involute profiles saves a lot of time that would otherwise be spent adjusting clamping eccentricity.

\section{CONCLUSIONS}

This paper draws the following conclusions.

- The measurement trajectory of involute profiles does not need to be changed if the clamping eccentric value is within $0.75 L$, but the profiles should be compensated to the default measurement position in the evaluation.

- The clamping eccentric error is caused by a coordinate transformation error between the workpiece coordinate system and the instrument coordinate system when the clamping eccentricity transformational vector $\beta_{i}$ is ignored.

- The eccentricity parameters obtained using the involute Model raised in this paper are consistent with those obtained using the Limacon model. Therefore, the eccentricity parameters estimation method proposed in this paper is correct and feasible.

- The clamping eccentric error compensation model compensates evaluation results to the state without eccentricity, allowing precision measurement of involute profile.

- The highest allowed clamping eccentric value can extend to $0.75 \mathrm{~L}$, which saves a lot of time otherwise spent in adjusting clamping eccentricity.

\section{ACKNOWLEDGEMENT}

This work was supported by the Technology Program of the Educational Office of Hei Longjiang Province, China (No.11553016)

\section{REFERENCES}

[1] Takeoka, F., Komori, M., Kubo, A., Fujio, H., Ito, T., Takatsuji, T., Osawa, S., Sato, O., Takeda, R. (2009). High-precision measurement of an involute artefact by a rolling method and comparison between measuring instruments. Measurement Science and Technology, vol. 20, no. 4, p. 1, DOI:10.1088/09570233/20/4/045105.

[2] Kiekbusch, T., Sappok, D., Sauer, B., Howard I. (2011). Calculation of the combined torsional mesh stiffness of spur gears with two-and threedimensional parametrical FE models. Strojniški vestnik - Journal of Mechanical Engineering, vol. 57, no. 11 , p. 810-818, DOI: $10.5545 / \mathrm{sv-}$ jme.2010.248.

[3] Lou, Z.F, Wang, L.D. (2010). Adjustment of the measurement point's position in a double-disc 
instrument for measuring an involute. Metrologia, vol. 47, no. 5, p. 583., DOI:10.1088/00261394/47/5/009.

[4] Frazer, R.C., Bicker, R., Cox, B., Harary, H., Härtig, F . (2004). An international comparison of involute gear profile and helix measurement. Metrologia, vol. 41, no. 1, p. 12-13, DOI:10.1088/0026-1394/41/1/003.

[5] Härtig, F., Kniel, K., Wäldele, F. (2004). High accurate gear measuring machine. VDI Berichte, vol. 1860, p. 409-415.

[6] Chen, H.Y, Ma, Y. (2011). Designed on fullclosed loop control system of gear measuring center. International Conference on Electronic \& Mechanical Engineering and Information Technology, p. 2015-2018.

[7] Jin, J.Q., D, L., Zhao, F.H. (2010). Measuring principle and technology of accumulate pitch error of large gear. The International Society for Optical Engineering Proceedings ICMIT, Gwangju, DOI:10.1117/12.858447.

[8] Li, W.L, Wei, L, Bai, L. (2008). On-machine measurement principle and measuring data treatment on large gears. The International Society for Optical Engineering Proceedings, Hefei, DOI:10.1117/12.819612.
[9] Yang, L, Sun, H.L, Yuan, H.J. (2011). A Study of a gear profile measuring method using gear measurement center. Strategic Technology (IFOST), 6th International Forum, p.366-369.

[10] Shi, X.M. (2009). Measurement and evaluation of involute cylindrical gear based on gear measurement center. $\mathrm{PhD}$ Thesis. Tianjin University, Tianjin (in Chinese).

[11] Tao L. (2010). Study on technology of offset error compensation of the workpiece in gear measurement. $\mathrm{PhD}$ Thesis. Harbin Institute of Technology, Harbin (in Chinese).

[12] ISO 1328-1:1995 (1997). Cylindrical gears-ISO systems of accuracy part 1: Definitions and allowable values of deviations corresponding flanks of gear teeth. International Organization for Standardization, Geneva.

[13] Alessandri, A., Cuneo, M., Pagnan, S., Sanguineti, M. (2007). A recursive algorithm for nonlinear least-squares problems. Computational Optimization and Applications, vol. 38, no. 5, p. 195-216, DOI:10.1007/s10589-007-9047-7.

[14] Murthy, T.S.R. (1986). A comparison of different algorithm for circularity evaluation. Precision Engineering, vol. 8, no. 6, p. 19-23, DOI:10.1016/0141-6359(86)90005-X. 